\title{
Use of hypotheses for analysis of variance models: challenging the current practice
}

\author{
Floryt van Wesel · Hennie Boeije • Herbert Hoijtink
}

(C) Springer Science+Business Media B.V. 2011

\begin{abstract}
In social science research, hypotheses about group means are commonly tested using analysis of variance. While deemed to be formulated as specifically as possible to test social science theory, they are often defined in general terms. In this article we use two studies to explore the current practice concerning group mean hypotheses. The first study consists of a content analysis of published articles where the reconstructed reality of hypotheses use is explored. The second study is a qualitative interview study with researchers, adding information about daily practice. We argue that, at present, hypotheses are not used to their utmost potential and that progress can be made by using informative hypotheses instead of the current non-informative hypotheses. Informative hypotheses capitalize on knowledge that researchers already possess and enable them to focus in their proceeding projects. The substantive focus of our work is the case of applied psychology.
\end{abstract}

Keywords ANOVA · Hypotheses · Informative hypotheses · Qualitative research

\section{Introduction}

When social scientists conduct research, they often have expectations about the social phenomenon that is being examined. Generally, such expectations are analyzed using statistical tests. However, when testing hypotheses most researchers use the traditional null and alternative hypothesis. In most cases, neither of these hypotheses contains information about the researchers' expectations and are therefore referred to as non-informative hypotheses. In contrast, informative hypotheses are expectations based on theory or common sense and are as specific as possible (Hoijtink et al. 2008). In this article we argue that research findings will be more accurate and thus provide a better answer to the research question if expectations are included in the statistical hypotheses. Accuracy is gained when such hypotheses are tested

F. van Wesel $(\varangle) \cdot$ H. Boeije $\cdot$ H. Hoijtink

Department of Methodology and Statistics, Utrecht University,

P.O. Box 80140, 3508 TC Utrecht, The Netherlands

e-mail: f.vanwesel@uu.nl 
straightforwardly instead of testing whether or not the non-informative null-hypothesis is rejected. The aim of this study is to investigate the current practice in order to assess the possibilities of using informative hypotheses instead of non-informative hypotheses.

We focus especially on research questions that involve differences between two or more groups (for instance, "Do researchers with different academic positions differ in their teaching skills?"). When testing an expectation or hypothesis following from such a research question concerning group means, analysis of variance (ANOVA) will usually be used. ANOVAs enable researchers to test whether or not the null-hypothesis can be rejected, usually at a $5 \%$ alpha level. In the current practice the null-hypothesis and the alternative hypothesis are represented by respectively $H_{0}$ : all group means are equal; there is no difference between the group means and $H_{A}$ : not all group means are equal; there is a difference between at least two of the group means. When $\mathrm{H}_{0}$ is rejected, it logically follows that the conclusion is $\mathrm{H}_{A}$ "not all group means are equal". The rejection of $\mathrm{H}_{0}$ is not considered a satisfying answer to the research question as it does not really involve the researcher's expectation, for instance that Ph.D. students will have fewer teaching skills than research fellows and full professors, except that we now know that not all group means are equal. We argue that the use of informative hypotheses can solve this issue.

An informative hypothesis may be formulated in terms of an ordering of the parameters (in this paper the group means) in which case it is called an (in)equality constrained hypothesis (Barlow et al. 1972; Silvapulle and Sen 2005; Hoijtink et al. 2008), or directional hypothesis. In this paper we make a distinction between simple order, main effect, and interaction effect informative hypotheses. Simple effect informative hypotheses are statements about expectations that involve the influence of one (categorical) independent variable on one (continuous) dependent variable. The effect is called simple because it only involves the ordering of single group means, for example: $\mathrm{H}_{\text {simple }}: \mu_{4}<\mu_{3}<\mu_{1}<\mu_{2}$, where $\mu_{j}$ denotes the population mean for group $j$. Main effect informative hypotheses are also statements about expectations that involve the influence of one (categorical) independent variable on one (continuous) dependent variable; these hypotheses involve the ordering of combinations of group means, for example:

$\mathrm{H}_{\text {main }}:\left(\mu_{1}+\mu_{3}\right) / 2>\left(\mu_{2}+\mu_{4}\right) / 2$. Interaction effect informative hypotheses are statements about expectations that involve the interaction between two or more (categorical) independent variables on one (continuous) dependent variable, for example:

$\mathrm{H}_{\text {interaction }}:\left|\mu_{1}-\mu_{3}\right|>\left|\mu_{2}-\mu_{4}\right|$, where $\left|\mu_{1}-\mu_{3}\right|$ represents the absolute difference between $\mu_{1}$ and $\mu_{3}$ (Wesel et al. submitted).

In order to investigate the potential for informative hypotheses in social science research, the current practice is investigated. Two studies are conducted: a content analysis of papers in peer-reviewed journals and a qualitative interview study involving researchers. In both studies we examine: What role do hypotheses play in the course of a social science research project? How do researchers presently formulate their expectations or hypotheses? Do researchers answer their research questions satisfactorily? Based on these results we discuss under what conditions the use of informative hypotheses will be beneficial with regard to the accuracy of the findings. The focus of the first study is on the reconstructed reality of hypotheses use described in research reports. In addition the second study will provide us with a description and understanding of the researchers' modus operandi with concern to the use of hypotheses. This is not necessarily equal to what usually is reported in terms of chronological order and activities.

As a substantive field we choose applied psychology. Since the context of this paper concerns ANOVA, we decided to search for a specialism within psychology where ANOVAs are likely to be used which led us to the specialized field of applied psychology. It is viewed that 
a reflection on the use of hypotheses is of relevance to this field since applied psychologists frequently use comparisons between groups.

\section{The first study: a content analysis}

This study involves a qualitative content analysis (Morgan 1993) of nineteen published applied psychology articles. This study was performed in order to explore:

(1) How hypotheses relate to the research goals and research questions

(2) If the proper analysis is used to test a hypothesis and to answer the research questions

(3) How hypotheses are formulated

\subsection{Method}

The articles were elected from three applied psychology journals: four articles from the Journal of Experimental Psychology: Applied, seven articles from the Journal of Applied Social Psychology and eight articles from the Journal of Applied Psychology. To decrease the number of appropriate articles we found, we included exclusively articles concerning a social or organizational psychology research topic which were published in 2007 or 2008 and which had a maximum length of 25 pages. Table 1 is an overview of the journals, the articles, the authors and the publication years.

Table 1 References and their numbers used in the content analysis per journal

\begin{tabular}{cl}
\hline No. & Reference \\
\hline Journal of Experimental Psychology: Applied \\
1 & Blanchette et al. (2007) \\
2 & Butler et al. (2007) \\
3 & Raghubir and Srivastava (2008) \\
4 & Wiener et al. (2007) \\
Journal of Applied Social Psychology \\
5 & Cremer et al. (2008) \\
6 & Einwiller and Kamins (2008) \\
7 & Goodman et al. (2008) \\
8 & Helweg-Larsen and LoMonaco (2008) \\
9 & McCulloch et al. (2008) \\
10 & Miron-Shatz and Ben-Shakar (2008) \\
11 & Yi-Feng et al. (2008) \\
Journal of Applied Psychology \\
12 \\
13 & Boezeman and Ellemers (2008) \\
14 & Cremer and Tyler (2007) \\
15 & Grant (2008) \\
19 & Gupta et al. (2008) \\
\hline & Heilman and Okimoto (2008) \\
17 & Wong and Anderson (2007) \\
19 & \\
\hline &
\end{tabular}


Table 2 Coding frame of content analysis of 19 articles in study 1 with frequencies (freq) of presence of codes, organized according to the three research questions

\begin{tabular}{|c|c|c|c|c|c|}
\hline \multicolumn{2}{|c|}{$\begin{array}{l}\text { How hypotheses relate to } \\
\text { research goals and } \\
\text { questions }\end{array}$} & \multicolumn{2}{|l|}{$\begin{array}{l}\text { Analysis of hypotheses } \\
\text { and research question } \\
\text { answered }\end{array}$} & \multicolumn{2}{|l|}{$\begin{array}{l}\text { How hypotheses are } \\
\text { formulated }\end{array}$} \\
\hline code & freq & code & freq & code & freq \\
\hline Basis of the research & & ANOVA use & & Type of effect & \\
\hline - Little/no research & 13 & - Intro. hypothesis test & 14 & - Interaction effect & 16 \\
\hline - Imperfect studies & 3 & - Manipulation check & 9 & - Main effect & 15 \\
\hline - Previous research & 1 & - Overall tests & 8 & Language used & \\
\hline - Improving studies & 1 & - Expectation test & 6 & - Inequalities & 15 \\
\hline Goal of the research & & - No hypothesis test & 6 & - Effect description & 13 \\
\hline - Expanding & 12 & - Use of post hoc test & 13 & - Mediator & 3 \\
\hline - Exploring & 8 & Contents of table & & - Moderator & 2 \\
\hline - Improving & 7 & - Descriptive statistics & 14 & & \\
\hline - Replicating & 5 & - Significance & 9 & & \\
\hline - Filling a gap & 2 & - Statistical tests & 4 & & \\
\hline \multirow[t]{4}{*}{ Expectation } & 18 & - Effect sizes & 2 & & \\
\hline & & Contents of figures & & & \\
\hline & & - Descriptive statistics & 8 & & \\
\hline & & - Interaction effects & 2 & & \\
\hline
\end{tabular}

To start a content analysis, a coding frame needs to be developed (Berg 2004). All articles dealing with certain themes were assigned the same code. We decided to construct a coding frame that was founded on the different article sections: introduction, method, analysis and conclusions. To fill in and, when necessary, to adjust this theoretical frame, we used open coding to start with; this is the process of examining, comparing, conceptualizing and categorizing data (Strauss and Corbin 1998). We worked our way through several articles until no new codes emerged. This occurred after six articles. The final coding frame allowed us to detect patterns and relationships between hypotheses and other parts of the papers such as the research questions and the analysis techniques used. Table 2 shows the final coding frame.

\subsection{Results}

Table 2 gives an overview of the numerical results. The values in this table are frequencies of the number of articles that were assigned a certain code. For example, it was found that in 13 out of 19 articles the research was legitimized by pointing to the absence of previous research on the topic.

\subsubsection{How hypotheses relate to research goals and questions}

The left panel of Table 2 shows the reasons mentioned for undertaking the research projects. The logic underlying most studies is that there is a paucity of research in a particular subject or that studies need to be improved because of imperfections. Most research aims to expand 
existing theories, to explore the field of study or to improve previous research. For example: "We present three studies that build upon and extend the social-identity based model of cooperation with the organization..." (2, p. 1013). The aim of expanding existing theories suggests that most hypotheses emerge partly from the existing literature. When exploring new fields, literature seems to be less important.

In most studies, hypotheses seem to be consistent with the theory that is used and with the research goals and questions, as in the following combination of an objective, a research question and a hypothesis:

Our mission expanded that of Higgens and Liberman, both by using other interventions and by examining whether these findings could be replicated with experts (15, p. 1275) The present study examines the efficacy of instructions to disregard preliminary information and interventions for reducing its effect on experts who regularly make hiring decisions (15, p. 1273)

Participants will disregard preliminary information when rating the candidates so that the ratings in the control condition will not vary according to whether the preliminary information was positive or negative. (15, p. 1276).

\subsubsection{Analysis of hypotheses and research question answered}

The articles in this study were selected based on their use of ANOVA to analyze the collected data. The results of our examination of the methods of analysis are presented in the middle panel of Table 2. In most of the articles the hypotheses are analyzed in a statistically appropriate manner although it is not always clear what hypotheses were tested, especially when the authors followed a more exploratory approach. This might be explained by the difference in exploratory and confirmatory research questions. We will follow up on this idea in Study 2.

Although the authors used appropriate statistical tests, it was not always clear whether the authors were able to answer their research questions. Take, for example, the hypothesis: "Applicants who were parents, whether mothers or fathers, would be rated as less likely to be committed to the job than would same sex applicants who were not parents" (10, p. 190). The authors expected a main effect for the variable "parental status" but, after analyzing a $2 \times 2$ ANOVA, they found a significant main effect both for sex and for parental status. As a follow-up procedure, the authors performed six post-hoc $t$-tests which indicated that all four groups differed (but not in what direction they differed). After describing these results, they neither concluded that their hypothesis was correct, nor that it was incorrect. Not all authors performed follow-up procedures and even when these were performed it was difficult to draw conclusions about the correctness of a hypothesis.

\subsubsection{How hypotheses are formulated}

As can be seen in the third panel of Table 2, most articles contained hypotheses about main effects as well as about interaction effects. It appears that researchers think about the relationships between the different group means in terms of (in)equalities, for example: "Disidentifiers will report a stronger intention to spread a negative rumor about the entity with which they disidentify than will identifiers, with neutral identifiers in the middle" (6, p. 2252). The reports indicate that the relationships between the group means are obvious in the case of main effects. They also indicate that interaction effect hypotheses are harder to formulate. This can be illustrated by the following example: The main effect hypothesis describes how all of the groups mentioned in the hypothesis relate to one another: 
When men and women are not provided with any gender stereotypical information about entrepreneurs, men will report stronger entrepreneurial intentions than women. $(9$, p. 1054).

The interaction effect hypothesis is not specific as it does not state anything about the relationship between men and women:

Respondent genders and stereotype activation will interact such that men will report stronger entrepreneurial intentions when presented with an implicit versus an explicit masculine stereotype whereas women will report stronger entrepreneurial intentions when presented with an explicit versus an implicit masculine stereotype. $(9$, p. 1055)

\subsection{Conclusions of the first study}

First of all, the researchers in the selected articles seem to follow the steps of the empirical cycle (Groot 1971) in reporting their research findings. Within this empirical cycle, the hypotheses take their natural place at the end of the deduction step and before data collection and analysis. Second, when researchers want to expand existing theories, their research approach may be more confirmatory because part of what they are studying has been investigated previously. Consequently, the hypotheses are used in a confirmatory manner. When researchers want to explore new fields, their research approach may be more exploratory because there is a scarcity of research on which to base their own research. Consequently, the hypotheses are used in an exploratory manner. Third, on the basis of the publications, we found that main effect hypotheses are easier to formulate than interaction effect hypotheses. Fourth, in most cases the hypotheses are tested to the best of the authors' abilities and, in general, conclusions are drawn based on the results of these tests. Fifth, translation of hypotheses and expectations into (in)equality constrained hypotheses seems to be justified since researchers already think about their expectations in terms of something being larger than, less than and equal to something else. This indicates that researchers are already familiar with ordering parameters, i.e. informative hypotheses, but that they do not use them to their utmost potential. Finally, the conducted research often does not provide a detailed answer to the research questions because the exact expectations are not included in $\mathrm{H}_{0}$ or $\mathrm{H}_{a}$ and are therefore not tested.

With the first study, insights were gained into the role of hypotheses in the reconstructed reality of published research reports. The second study elaborates on the findings above and puts them further into perspective in the actual practice of psychological research.

\section{The second study: interviews}

The interview study involves semi-structured interviews with fourteen applied psychology researchers. Three research questions are examined:

(1) How do researchers commonly generate their hypotheses?

(2) What role do hypotheses play during a research project?

(3) What are the advantages and disadvantages of the modus operandi and informative hypotheses? 


\subsection{Method}

A purposive sample, composed of applied researchers working at several Dutch universities, was used to collect information from different research disciplines within the field of psychology. We chose different characteristics that were thought to be related to the use of hypotheses since we were interested in the range of different views and practices. The sample consisted of six female and eight male psychologists. Furthermore, two participants were $\mathrm{PhD}$ candidates, one was a Post Doc, six were assistant professors and five were full professors. Of these participants, five researchers mainly conducted survey research and nine researchers mainly conducted experiments.

We chose semi-structured interviews in order to gain more in-depth information about the researchers' perspectives on conducting research. The instrument used was constructed based on the research questions as described above and on the findings of content analysis. This resulted in a topic list which is found in the Appendix. Probes were used if answers needed further exploration or clarification. All interviews were conducted in Dutch by the same interviewer. Confidentiality was discussed before the interviews started. All interviews were recorded and transcribed. Three pilot interviews were conducted before interviewing the sampled researchers in order to refine the topic list and to train interviewing skills.

Interviews were analyzed using MAXQDA2007, software for qualitative data analysis. A thematic analysis was conducted consisting of open, axial and selective coding (Boeije 2010; Strauss and Corbin 1998). First, open coding was applied separately to each interview. After fourteen interviews, no new codes emerged and data collection was finalized. The second phase in the analysis was that of axial coding, whereby we defined and demarcated categories determined their relevance and reorganized the codes. We ended the analysis after selective coding, which is intended to integrate the categories into a conceptual model.

\subsection{Results}

The analysis of the interview data led to the model shown in Fig. 1. The boxes represent the main concepts that emerged during the analysis. The relationships between the main concepts, as described by the participants, are represented by the solid arrows. Since most participants were ignorant about informative hypotheses, they were not able to discuss their experiences using them. However, they reflected on their possible future use. For this reason, the concept "Informative hypotheses" is represented by an oval and the described relationships are represented by the dashed arrows.

\subsubsection{The generation of hypotheses}

Grey area A in Fig. 1 contains the main concepts concerning the generation of hypotheses. The participants reported that the most important source for hypotheses generation is literature. In addition to literature, common sense, their own previous research projects and meetings with colleagues were also frequently mentioned:

Hypotheses can be formed based on literature, but they can also originate in our own previous research, completing that research topic. Or, they can arise in conversations between my $\mathrm{PhD}$ candidates and me. (resp.11, male, professor).

The researchers noted two moments at which hypotheses were formulated: One came after studying the literature and writing the introduction of the draft report and the other 


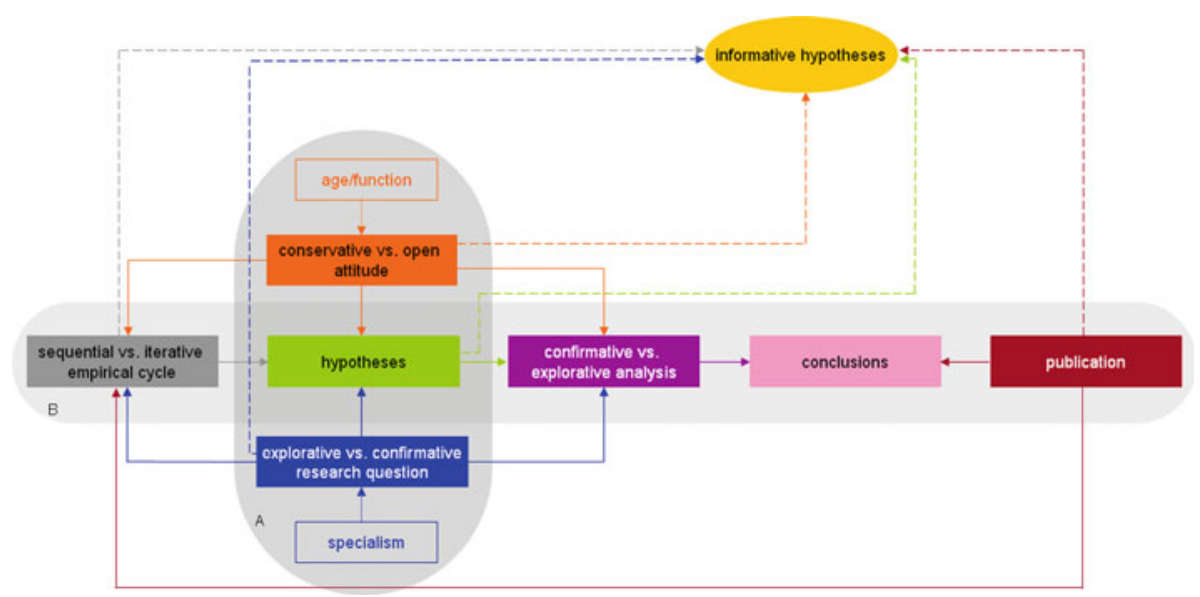

Fig. 1 Model describing relationships between main concepts in study 2

came after analyzing the collected data, when hypotheses are further specified. The interviewees explained that when the results of a study conflicted with the expectations stated in the introduction, the hypotheses needed to be (re)formulated in order to get a paper published.

Similarly to what we found in the first study, all of the researchers indicated that when formulating hypotheses they think in terms of (in)equalities independent of type of effect (simple, main or interaction). In addition, they said that interaction effect hypotheses are harder to formulate than simple and main effect hypotheses. For example:

In most cases I know what the interaction effect looks like, but when there are more than two factors, it gets complicated. (resp.11, male, professor).

The process of hypothesis generation is influenced by the attitude of the research group and by the type of research questions someone is interested in (see grey area A in Fig. 1). In our study, the researcher's attitude towards conducting research can either be conservative, meaning that the empirical cycle will be followed precisely and that field specific customs indicate how research is conducted, or open, meaning that the rules of the empirical cycle are flexibly interpreted and that new research methods are welcome to the field. More conservative researchers tend to formulate their hypotheses early on in the research project and the hypotheses then dominate the research design, which is implied by the empirical cycle. In addition, the researchers' attitudes seem to be influenced by their age and function, in the sense that older researchers and researchers with higher positions tend to be more conservative than younger researchers.

Research questions can be either more strongly confirmatory or more strongly exploratory. A confirmatory research question (when the goal is to expand) seems to lead to the specification of hypotheses early on in the research project, whereas an exploratory research question (when the goal is to explore) seems to lead to the formulation of hypotheses after the data are analyzed, - if they are formulated at all. An example of formulating hypotheses later on in the project is:

This research was more exploratory, I wanted to see what happened... After the paper was finished, my supervisor asked me if it might be an idea to insert some hypotheses afterwards. (resp.2, female, assistant prof.) 
We found indications that a psychologist's specialization may influence whether a research question is confirmatory or exploratory. Specialization, in this case, is a specific part of the general research field psychology, such as social psychology. A different specialism may have a different research tradition.

\subsubsection{Role of hypotheses during the research project}

Grey area B in Fig. 1 contains the concepts that the interviewees relate to the role of the hypotheses in their research projects. The core concepts of this area all refer to a stage of the empirical cycle.

The interviewed researchers indicated that if they had hypotheses before data collection, the analysis was confirmatory. This means that the hypotheses and model assumptions were tested first, and that their conclusions were directly linked to the hypotheses. In this scenario, hypotheses play a leading role in the research project. One of the participants said:

First, I always test the main effects, to replicate previous findings, and then I add the moderating variables. In this way I systematically test the theory. (resp.13, male, professor).

When hypotheses were absent, the analysis was exploratory, meaning that several tests were performed and that conclusions were drawn based on what interesting significant results appeared during the analysis. In this scenario, the hypotheses, in most cases added to the introduction after the analysis was done or because a reviewer asked for them, only play a minor role, if any. Both of these scenarios can be influenced by attitude and research question. More conservative researchers tend to work with hypotheses and prefer a confirmatory analysis, and more open researchers tend to work without hypotheses and prefer an exploratory analysis. An explorative research question leads to an explorative analysis approach and a confirmative question leads to a confirmative analysis approach.

A process found to be linked to the above is a sequential versus an iterative use of the empirical cycle. We observed two ways of using the empirical cycle. A sequential use implies that the stages and corresponding rules (introduction ending with hypotheses, methods, analysis, conclusion and discussion) are being strictly followed. An iterative use implies that researchers jump from one stage of the cycle to another, revisiting stages that have already been conducted. An example of the latter is:

When the results are opposite to what we expected, we need to see what we can make of it. Can we find corresponding literature? Or, how can we turn our story around so it all fits together? (resp. 10, male, assistant prof.).

Here we noticed that a conservative attitude and/or a confirmatory research question resulted in a preference for the sequential way, whereas an open attitude and/or an exploratory research question resulted in a preference for the iterative way.

There is one activity dominating the entire research process: publication (right hand side of Fig. 1). Our definition of publication includes everything related to the publishing of the report of a research project in the form of a paper in a scientific journal. The following quotes will illustrate various aspects of conducting research that are influenced by the current practice of publishing psychological research:

Introduction: $\mathrm{PhD}$ students don't have that much time to spend on exploring the literature... They are in a constant hurry. (resp. 4, male, professor). 
New methodology: In our field we have certain traditions and I am not someone who challenges the current rules because it can slow down the publication of the paper. (resp. 11, male, professor).

Analysis: At some point what is right and wrong and what you can and cannot do is not the question, it is do you get away with it by the reviewers... as long as the reviewer does not complain, it is fine. (resp. 6, female, prof. assistant).

Results: Originally, this picture was in the paper... Journals don't want too many pictures, although it makes clear what the effect looks like. (resp. 5, male, assistant prof).

\subsubsection{Advantages and disadvantages of informative hypotheses versus modus operandi}

The third research question of this second study explores the advantages and disadvantages experienced in the current practice and a reflection on the future of informative hypotheses. The dashed arrows in Fig. 1 represent the relationships between the concepts previously discussed and the use of informative hypotheses. The relationships that were found can be seen as an indication of the situations in which informative hypotheses can be an improvement over the modus operandi and under what circumstances researchers will actually use them.

Advantages of informative hypotheses over ANOVAs with follow-up tests mentioned by the interviewed researchers were: progress in analysis techniques for the field, the possibility to test very specific theories, progress in knowledge building, forcing researchers to think more thoroughly about their research and opening up new possibilities (meaning that certain interaction effect hypotheses can be tested):

We wanted to look at certain interaction effects but we couldn't figure out what the correct contrasts should be as we wanted to test the whole interaction effect at once. We were bound by the possibilities of ANOVAs with follow-up tests. With your technique, that would have been possible. (resp. 7, female, assistant prof.)

Disadvantages that were mentioned were that the researchers were content with the current modus operandi, that they lacked the knowledge and skills to learn the technique, that specifying informative hypotheses would be too time-consuming, that formulating informative hypotheses is a subjective technique and that the researchers feared that it would be difficult to get such an article published. To emphasize this, one of the participants said:

The problem with researchers is that they have to publish their work. They need to find journals that accept this kind of new unknown techniques. (resp.5, male, professor).

The participants were more inclined to use informative hypotheses when they had an open attitude, but they were aware that they needed a confirmatory research question, that they should formulate their hypotheses before data collection and that they should use the empirical cycle in a sequential way.

\subsection{Conclusions of the second study}

Most of the results we found in the interview study correspond with the results of the content analysis of the published papers. First, hypotheses commonly originate in literature and/or common sense. Second, interaction effect hypotheses are harder to formulate then main effect hypotheses. Third, researchers naturally think about group means in terms of (in)equalities: This can be considered an argument for using informative hypotheses. Apart from these similarities, we found reports and practice diverging on several accounts. First, in analyzing the 
interviews we found that the hypotheses play an important role in a research project when the researchers have a conservative attitude, a confirmative research question and when they follow the empirical cycle. The hypotheses play a minor role when the researchers have an open attitude, an explorative research question and when they do not follow the empirical cycle. Second, the data suggest that psychologists conducting experiments generally choose a more confirmative approach, whereas psychologists using surveys generally use a more explorative approach. Third, we found that the introduction of informative hypotheses to applied researchers may be problematic. Researchers who have a more open attitude are willing to use informative hypotheses but they do not benefit especially from using them because they generally have explorative research questions.

\section{Discussion and recommendations}

In the two studies we conducted, we investigated how hypotheses concerning group means are used in reported social science research and in daily practice. Results from both studies concurred when they pertained to how hypotheses are formulated: (1) Hypotheses are formulated using phrases like "one group will have a higher score than another group", (2) Main effect hypotheses are easier to formulate than interaction effect hypotheses. The second finding is in agreement with literature on explicit and tacit knowledge (Polanyi 1966; Sanders 1988). Explicit knowledge is knowledge that people actively possess and of which they are aware. Tacit knowledge is knowledge that people are not aware of possessing but that they are nevertheless able to use. Within this context, main effect hypotheses reflect explicit knowledge as they express the more obvious relationships between the group means and they originate in literature and in what is already known. Interaction effect hypotheses reflect tacit knowledge as they concern thinking more thoroughly about the ordering and deciding on what would be the most obvious expectation. The results of the two studies differed in the actual use of the hypotheses. One such difference is that not all steps taken in research practice are reported, leaving the readers of the articles unaware of, for example, non-significant results, changes in research methods or experimental settings, and changes in what was expected or hypothesized.

The core question of this paper is if researchers in social science research are able to answer their research questions in a satisfying way by means of their hypotheses. We found that, on the one hand, psychology researchers do the best they can, using the statistical techniques they have mastered over time; they indicate that they are quite satisfied with the answers they get concerning their research questions. On the other hand, they do not seem to be aware of getting very little information about the correctness of their hypotheses and thus that only a part of their research question is answered.

Results from this research indicate that using informative hypotheses instead of non-informative hypotheses is a relatively small step to take that can lead to a considerable improvement in research question answers: When researchers evaluate their existing specific theoretically founded hypotheses instead of default non-informative hypotheses they will get more accurate research findings and thus to better answers to their research questions. Furthermore, formulating informative hypotheses enables researchers to use their existing knowledge as a basis for generating new knowledge and it may refine their research goals and research questions, clarifying the aims of their research projects.

Of course, both studies described above have their limitations. For both studies, generalization is very limited, due to the selective and small samples. As there is a clear format for publishing psychological research, it is not surprising that, in the content analysis, we found 
Box 1 Recommendations for using informative hypotheses

- Use informative hypotheses when you have a confirmatory research question rather than when you have an exploratory research question.

- $\quad$ Specify your expectations after studying the literature and before collecting data.

- Invest time and energy in formulating the informative hypotheses properly.

- Use multiple sources on which to base expectations. As the informative hypotheses determine the analysis results, they should originate in a broad evidence base.

- $\quad$ Analyze the hypotheses with, for instance: Post-hoc tests (Klockars and Sax 1986; Toothaker 1993), planned contrasts (Rosenthal et al. 2000), Constrained Statistical Inference (CSI) (Barlow et al. 1972; Robertson et al. 1988; Silvapulle and Sen 2005), the Order Restricted Information Criterion (ORIC, Anraku 1999), and Bayesian model selection for (in)equality constraint hypotheses (Hoijtink et al. 2008; Mulder et al. 2009; Wesel et al. 2010).

- Find more information about the possibilities of informative hypotheses and analysis software on: http://tinyurl.com/informativehypotheses.

consistency in research goal, research question, hypotheses and conclusions, since these articles are already peer-reviewed whereby fatal flaws are less likely to occur. As most of the researchers who participated in the interview study were not acquainted with informative hypotheses, it is difficult to study the practical implications of using informative hypotheses instead of non-informative hypotheses.

Recommendations for social science researchers who are willing to try a new research approach can be found in Box 1 .

Two major issues prevent researchers from changing their modus operandi: Attitude towards research and attitude towards publication. Concerning the first, the information obtained from the interviews indicates that researchers who can benefit most from using informative hypotheses (researchers who have a confirmatory research question) are less likely to use them because they generally have a more conservative attitude towards conducting research. Concerning the latter, when articles that use new methods are not published, progress in a scientific field will stagnate. Therefore, we recommend that editors and reviewers keep an open mind when new methods are presented in an applied article.

\section{Appendix}

The topic list that was used for the interviews of the second study

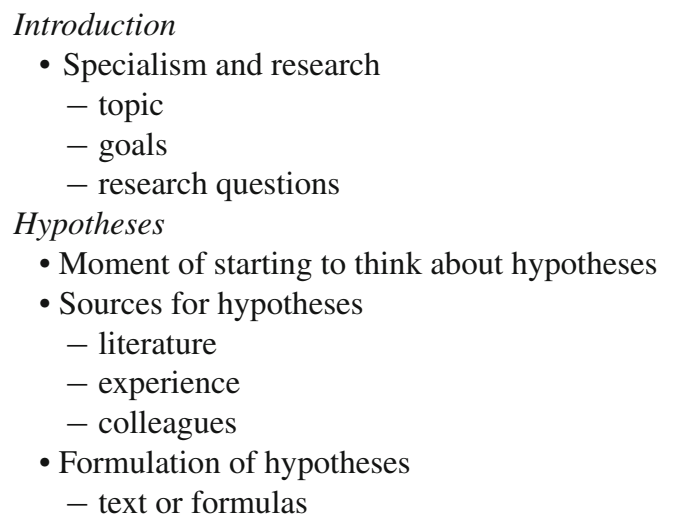


- type of effect (main, interaction)

- links with analysis

- Formulating interaction effects

- Effect sizes

- Standard null and alternative hypotheses

- Test what you hypothesized

- Limitations

- Satisfaction

Analysis

- Analysis procedure

- Reasons for testing

- Post hoc tests/planned contrasts

- reasons for use

- conclusions

- Satisfaction

- statistical techniques

- own knowledge

\section{Conclusions}

- Drawing conclusions

- Satisfaction

- Reporting habits

Informative Hypotheses

- Analysis techniques

- Advantages

- Disadvantages

- Deviate hypotheses

- Promoting informative hypotheses

\section{References}

\section{References marked with an * are used in the content analysis.}

Anraku, K.: An information criterion for parameters under a simple order restriction. Biometrica 86, 141-152 (1999)

Barlow, R., Bartholomew, D., Bremner, J., Brunk, H.: Statistical inference under order restrictions. Wiley, New York (1972)

Berg, B.L.: Qualitative research methods for the social sciences. Pearson Education Inc., Boston (2004)

*Blanchette, I., Melnuk, L., Richards, A., Lavda, A.: Reasoning about emotional contents following shocking terrorist attacks: a tale of three cities. J. Exp. Psychol. 13, 47-56 (2007)

Boeije, H.: Analysis in qualitative research. Sage, London (2010)

*Boezeman, E., Ellemers, N.: Volunteer recruitment: the role of organizational support and anticipated respect in non-volunteer organizations. J. Appl. Psychol. 93, 1013-1026 (2008)

*Butler, A., Karpicke, J., Roediger, H.: The effect of type and timing of feedback on learning from multiplechoice tests. J. Exp. Psychol. 13, 273-281 (2007)

*de Cremer, D., van Knippenberg, D., van Dijk, E., Leeuwen, E. van.: Cooperating if one's goals are collective-based: social identification effects in social dilemmas as a function of goal transformation. J. Appl. Soc. Psychol. 38, 1562-1579 (2008)

*de Cremer, D., Tyler, T.: The effects of trust in authority and procedural fairness on cooperation. J. Appl. Psychol. 92, 639-649 (2007)

*Einwiller, S., Kamins, M.: Rumor has it: the moderating effect of identification on rumor impact and the effectiveness of rumor refutation. J. Appl. Soc. Psychol. 38, 2248-2272 (2008) 
*Goodman, J., Schell, J., Alexander, M., Eidelman, S.: The impact of a derogatory remark on prejudice toward a gay male leader. J. Appl. Soc. Psychol. 38, 542-555

*Grant, A.: The significance of task significance: job performance effects, relational mechanisms, and boundry conditions. J. Appl. Psychol. 93, 108-124 (2008)

Groot, A.D.: Methodologie, grondslagen van onderzoek en denken in de gedragswetenschappen. Mouton en Co, 's-Gravenhage (1971)

*Gupta, V., Bhawe, N., Turban, D.: The effect of gender stereotype activation on entrepreneurial intentions. J. Appl. Psychol. 93, 1053-1061 (2008)

*Heilman, M., Okimoto, T.: Motherhood: a potential source of bias in employment decisions. J. Appl. Psychol. 93, 189-198 (2008)

*Helweg-Larsen, M., LoMonaco, B.: Queuing among u2 fans: reactions to social norm violations. J. Appl. Soc. Psychol. 38, 2378-2393 (2008)

Hoijtink, H., Klugkist, I., Boelen, P.A. (eds.): Bayesian evaluation of informative hypotheses. Springer, (2008)

*Klehe, U.-C., Anderson, N.: Working hard and working smart: motivation and ability during typical and maximum performance. J. Appl. Psychol. 92, 978-992 (2007)

Klockars, A., Sax, G.: Multiple comparisons. Sage university paper series on quantitative applications in the social sciences. SAGE, CA (1986)

*Lievens, F., Sanchez, J.: Can training improve the quality of inferences made by raters in competency modeling? A quasi-experiment. J. Appl. Psychol. 92, 812-819 (2007)

*McCulloch, K., Albarracin, D., Durantini, M.: A door to HIV-prevention interventions: how female-targeted materials can enhance female participation. J. Appl. Soc. Psychol. 38, 1211-1229 (2008)

*Miron-Shatz, T., Ben-Shakar, G.: Disregarding preliminary information when rating job applicants' performance: mission impossible? J. Appl. Psychol. 38, 1271-1294 (2008)

Morgan, D.L.: Qualitative content analysis: a guide to paths not taken. Qual. Health Res. 3, 112-121 (1993)

Mulder, J., Hoijtink, H., Klugkist, I.: Inequality and equality constrained multivariate linear models: objective model selection using constrained posterior priors. Stat. Plan. Inference 140, 887-906 (2009)

Polanyi, M.: The tacit dimension. Doubleday \& Co, New York (1966)

*Raghubir, P., Srivastava, J.: Monopoly money: the effect of payment coupling and form on spending behavior. J. Exp. Psychol. 14, 213-225 (2008)

Robertson, T., Wright, F.T., Dykstra, R.L.: Order restricted statistical inference. Wiley, New York (1988)

Rosenthal, R., Rosnow, R.L., Rubin, D.B.: Contrasts and effect sizes in behavioral science: a correlational approach. Cambridge University Press, Cambridge (2000)

Sanders, A.: Michael Polanyi's post critical epistemology, a reconstruction of some aspects of 'tacit knowing'. Rodopi, Amsterdam (1988)

Silvapulle, M., Sen, P.: Constrained statistical inference. Wiley, Hoboken (2005)

Strauss, A., Corbin, J.: Basics of qualitative research, techniques and procedures for developing grounded theory. Sage, Thousand Oaks (1998)

Toothaker, L.: Multiple comparisons procedures. Sage University paper series on quantitative applications in the social sciences. SAGE, Thousand Oaks (1993)

van Wesel, F., Hoijtink, H., Klugkist, I.: Choosing priors for inequality constrained normal linear models: methods based on training samples. Scand. J. Stat. (2010) doi:10.1111/j.1467-9469.2010.00719.x

van Wesel, F., Klugkist, I., Hoijtink, H.: Formulating and analyzing interaction effects (submitted)

*Wiener, R., Holtje, M., Cantone, J., Winter, R., Gross, K.: Consumer credit card use: the roles of creditor disclosure and anticipated emotion. J. Exp. Psychol. 13, 32-46 (2007)

*Wong, K., Kwong, J.: Effects of rater goals on rating patterns: evidence from an experimental field study. J. Appl. Psychol. 92, 577-585 (2007)

*Yi-Feng, N. C., Peiguan, W., Tjosvold, D.: Effects of relationship values and goal interdependence on guanzi between foreign managers and Chinese employees. J. Appl. Soc. Psychol. 38, 2440-2468 (2008) 